\title{
Effect of Environmental Hypothermia on Dorsal Aortic Blood Flow in the Chick Embryo, Stages 18 to 24
}

\author{
JON WISPE, NORMAN HU, AND EDWARD B. CLARK ${ }^{(21)}$ \\ Division of Pediatric Cardiology, Department of Pediatrics and the Cardiovasclar Center, University of Iowa \\ Hospitals and Clinics, Iowa City, Iowa USA
}

\begin{abstract}
Summary
Dorsal aortic blood flow was measured with a pulsed-Doppler meter in Hamburger-Hamilton stage 18, 21, and 24 chick embryos, and stroke volume index was calculated by dividing mean blood flow per minute by heart rate. These parameters were measured at baseline temperature $34.7^{\circ} \mathrm{C}$ after cooling to $31.1^{\circ} \mathrm{C}$ and subsequent rewarming to $34.2^{\circ} \mathrm{C}$. In stage 21 embryos, after environmental cooling, heart rate decreased from $170 \mathrm{bpm}$ to 118 bpm $(P<0.01)$, mean dorsal aortic blood flow decreased from $0.38 \mathrm{~mm}^{3} / \mathrm{sec}$ to $0.24 \mathrm{~mm}^{3} / \mathrm{sec}(P<0.01)$ but stroke volume index did not change [baseline, $0.13 \mathrm{~mm}^{3} /$ beat; after cooling, $0.12 \mathrm{~mm}^{3} /$ beat; after rewarming, $0.15 \mathrm{~mm}^{3} /$ beat $(P=$ N.S.)]. Similar results were observed in stage 18 and 24 embryos. The bradycardic response to environmental hypothermia was independent of functional autonomic innervation and probably mediated by a direct suppression of cell action potential dv/dt. Myocardial cell function was not adversely affected by an acute change in environmental temperature as the index of stroke volume was not altered, and all parameters returned rapidly to baseline with rewarming.
\end{abstract}

A change in body temperature alters cardiovascular function in man and animals. Heart rate, blood pressure, and cardiac output decrease with hypothermia $(6,17)$. These phenomena have been studied extensively in adult animals because of their importance in accidental and medical hypothermia (12). The mechanism responsible for bradycardia after a decrease in body temperature is unclear. The results of animal experiments suggest that the mechanism operates directly at the level of the heart; however, previous studies are confounded by the effects of anesthesia, shivering, and autonomic innervation (17).

We hypothesize that the slowing of heart rate is a basic response independent of functional autonomic innervation or a discrete cardiac conducting system. We studied this response in the chick embryo at early stages of cardiac development, and observed that stroke volume remained constant whereas heart rate and cardiac output decreased directly with environmental temperature.

\section{MATERIALS AND METHODS}

Fertile white Leghorn chicken eggs were incubated blunt-end up in a forced-draft constant humidity incubator to HamburgerHamilton (9) stages 18 (approximately 3 days), 21 (approximately $3 \frac{1}{2}$ days) and 24 (approximately 4 days). An egg was removed from the incubator and positioned on a stage surrounded by a warm air curtain at $38^{\circ} \mathrm{C}$. We opened the shell and removed the outer and inner shell membranes to expose the embryo. We measured the diameter of the dorsal aorta at the level of the sinus venosus using a filar micrometer eyepiece, calibrated against a 10-micron scribed glass standard.

Environmental temperature was monitored with a $1-\mathrm{mm}$ thermistor probe (Omega, Inc.) placed adjacent to the embryo. Although it was not technically possible to measure embryo body temperature, we assumed core temperature was directly related to environmental temperature. We recognize, however, that these temperatures may differ because of heat produced by metabolism or stored in the yolk.

We measured the velocity of blood flow through the dorsal aorta with a pulsed-Doppler velocity flow meter (1). The Doppler probe is a 1-mm diameter piezoelectric crystal mounted in epoxy at the tip of an 18-gauge needle. The crystal was positioned using a protractor jig at a $45^{\circ}$ angle over the dorsal aorta at the level of the sinus venosus (Fig. 1). At a constant angle between the crystal and blood vessel, the difference between transmitted and received frequency (Doppler shift) is linearly related to blood velocity. In vitro measurement in our laboratory demonstrates a linear relationship between measured and actual velocity over a range of $0-16 \mathrm{~mm}^{3} / \mathrm{sec}\left(\mathrm{y}=0.85 \mathrm{x}+1.18, r^{2}=0.99\right.$, S.E.E. $=0.49 \mathrm{~mm}^{3} /$ sec) (1). We recorded phasic and electrically integrated mean dorsal aortic blood velocity and computed heart rate by counting phasic pulsations between time lines. Mean dorsal aortic blood flow $(\bar{Q})$ was calculated from the equation: $\bar{Q}=\bar{V}_{\pi} \mathrm{d}^{2} / 4$ where $\overline{\mathrm{V}}$ is mean dorsal aortic blood velocity and $\mathrm{d}$ is dorsal aortic diameter. The index of stroke volume, dorsal aortic blood flow per cardiac cycle, was calculated by dividing mean dorsal aortic blood flow per minute by heart rate.

After baseline recording, we pipetted $0.5 \mathrm{ml}$ of $20^{\circ} \mathrm{C}$ chick Ringer's lactate on the embryo. We allowed $5 \mathrm{~min}$ for the temperatures of the fluid and embryo to reach an equilibrium. We recorded dorsal aortic velocity and heart rate. The fluid surrounding the embryo was rewarmed to baseline temperature over a 10 -min period by directing $38^{\circ} \mathrm{C}$ warm air flow at the egg. The environmental temperature, heart rate, and dorsal aortic blood velocity were again recorded.

We also studied two groups of control embryos. We measured the hemodynamic response to normothermic chick Ringer's lactate in the first group. Dorsal aortic blood velocity and heart rate were recorded before and after applying $0.5 \mathrm{ml} 38^{\circ} \mathrm{C}$ chick Ringer's lactate to the embryo. In the second control group, we measured dorsal aortic diameter before and after applying $20^{\circ} \mathrm{C}$ chick Ringer's lactate to assess the effect of temperature on aortic dimension.

The data are presented as mean \pm S.E.M. We used the paired $t$ test to analyze temperature effect on aortic diameter and the response to normothermic Ringer's lactate. Statistical analysis of the experimental embryos was carried out using one-way analysis of variance and Tukey's HSD test. Probabilities of less than $1 \%$ $(P<0.01)$ are presented as statistically significant. 


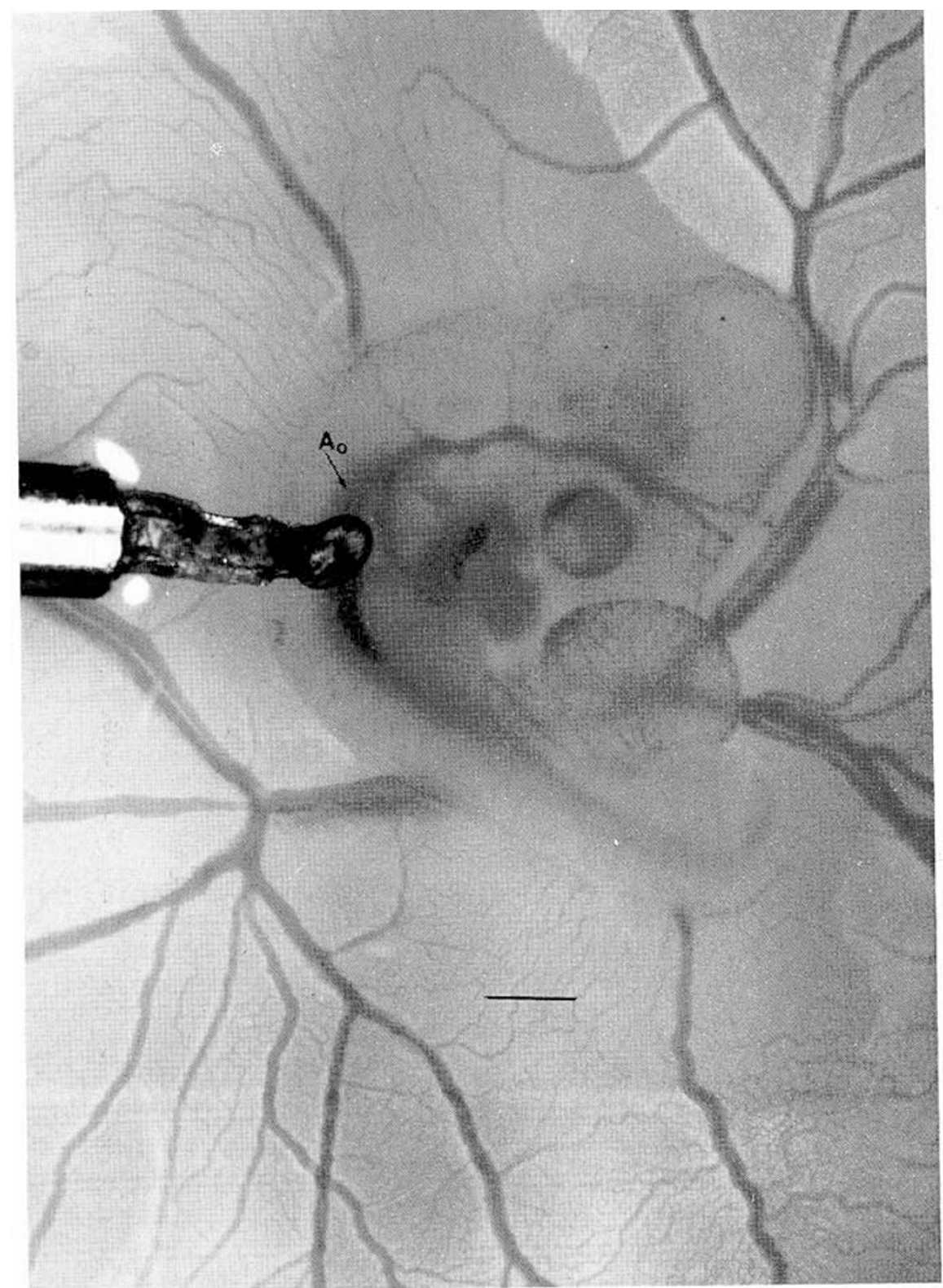

Fig. 1. Photomicrograph of stage 21 chick embryo. The $1-\mathrm{mm}$ piezoelectric crystal is shown positioned over the dorsal aorta (Ao). Bar is $1 \mathrm{~mm}$ $(\times 11)$.

\section{RESULTS}

No change was observed in aortic diameter, heart rate or dorsal aortic blood flow among the control groups (Tables 1 and 2). Although mean heart rate increased with the stage of development, the difference was not statistically significant. Among the experimental embryos, we found a direct relationship between heart rate and environmental temperature (Fig. 2). Heart rate decreased significantly in response to environmental cooling and increased to baseline with rewarming. The change in heart rate among experimental embryos at stages 18,21 , and 24 was similar.

Mean dorsal aortic blood flow was directly proportional to developmental stage (Fig. 3). The differences among the baseline blood flows reflected the 2-fold increase in embryo mass between stages. Dorsal aortic blood flow decreased significantly with environmental cooling and returned to baseline with rewarming; however, the stroke volume index remained unchanged and independent of environmental temperature (Fig. 4).

\section{DISCUSSION}

The effect of temperature on cardiac function is studied extensively in adult mammals. Cardiac output and heart rate decrease with a reduction in body temperature and have similar temperature coefficients (17). Investigators report that stroke volume remains constant in spite of a decrease in cardiac output and heart rate $(12,17)$. Three factors confound hypothermic studies in adult animals: $(1)$ anesthetic drugs blunt the response to a lower body temperature; (2) the animal shivers, thus producing metabolic heat; and (3) the autonomic nervous system also alters the heart rate and cardiac output (17).

We avoided these complications by studying the response of the embryonic heart to environmental hypothermia. No anesthetic drug was used and the chick embryo did not shiver. The autonomic nervous system is still developing at the stages studied. Branches of the vagus nerve are present in the ventricle at stage 24 , but functional cholinergic innervation does not occur unti] stage 38 (13). Sympathetic nerve fibers appear in the myocardium 
Table 1. Effect of $0.5 \mathrm{ml} 37^{\circ} \mathrm{C}$ chick Ringer Lactate on hemodynamic parameters in stage 18,21 , and 24 chick embryo, $(\bar{X} \pm$ S.E.M.) (S.V.I., stroke volume index)

\begin{tabular}{|c|c|c|c|c|}
\hline & $\begin{array}{c}\mathrm{T} \\
\left({ }^{\circ} \mathrm{C}\right)\end{array}$ & $\begin{array}{c}\mathrm{HR} \\
(\mathrm{bpm})\end{array}$ & $\begin{array}{c}\overline{\mathrm{Q}} \\
\left(\mathrm{mm}^{3} / \mathrm{sec}\right)\end{array}$ & $\begin{array}{l}\text { S.V.I. } \\
\left(\mathrm{mm}^{3}\right)\end{array}$ \\
\hline \multicolumn{5}{|c|}{ Stage $18(n=10)$} \\
\hline Before & $35.8 \pm 0.4$ & $173 \pm 4$ & $0.27 \pm 0.02$ & $0.09 \pm 0.01$ \\
\hline$P$ & N.S. & N.S. & N.S. & N.S. \\
\hline \multicolumn{5}{|c|}{ Stage $21(n=8)$} \\
\hline Before & $35.3 \pm 0.4$ & $169 \pm 4$ & $0.41 \pm 0.04$ & $0.15 \pm 0.01$ \\
\hline \multicolumn{5}{|c|}{ Stage $24(n=10)$} \\
\hline Before & $36.3 \pm 0.1$ & $190 \pm 4$ & $0.61 \pm 0.03$ & $0.20 \pm 0.01$ \\
\hline After & $35.8 \pm 0.3$ & $184 \pm 5$ & $0.63 \pm 0.03$ & $0.21 \pm 0.01$ \\
\hline$P$ & N.S. & N.S. & N.S. & N.S. \\
\hline
\end{tabular}

Table 2. Effect of environmental temperature on aortic diameter, $(\bar{X} \pm$ S.E.M. $)$

\begin{tabular}{lcc}
\hline & $\begin{array}{c}\text { Temperature } \\
\left({ }^{\circ} \mathrm{C}\right)\end{array}$ & $\begin{array}{c}\text { Ao diameter } \\
(\mathrm{mm})\end{array}$ \\
\hline $\begin{array}{l}\text { Before applying } 20^{\circ} \mathrm{C} \\
\text { chick Ringers }\end{array}$ & $34.2 \pm 0.4$ & $0.37 \pm 0.1$ \\
$\begin{array}{c}\text { After applying } 20^{\circ} \mathrm{C} \\
\text { chick Ringers }\end{array}$ & $31.3 \pm 0.8$ & $0.37 \pm 0.1$ \\
$P \quad<0.01$ & N.S. \\
\hline
\end{tabular}

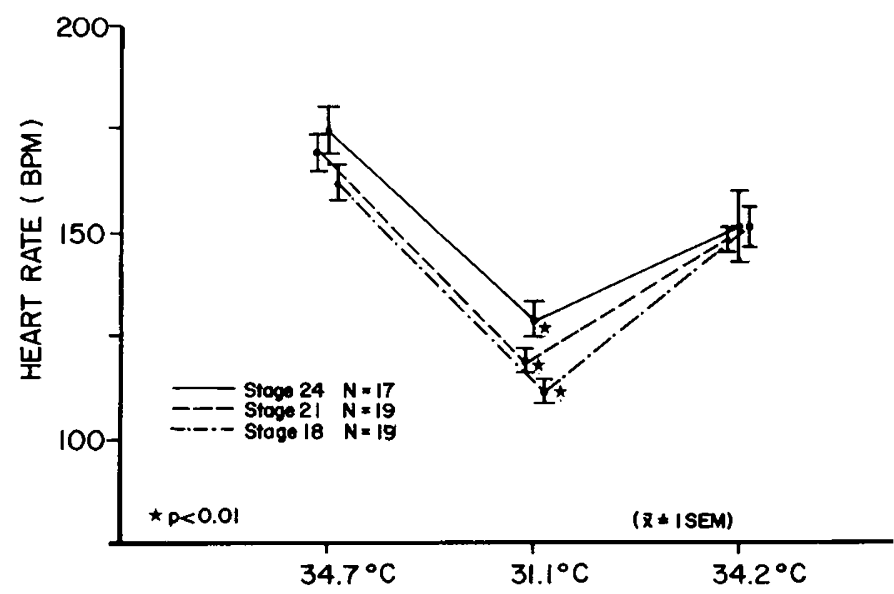

Fig. 2. Graph of heart rate versus environmental temperature for experimental embryos at stages 18,21, and 24 .

at stage 36 , but functional adrenergic nerve transmission begins just before hatching $(4,7,10)$; thus, functional autonomic innervation of the heart occurs after the stages studied in our experiments.

Defining the effect of temperature on cardiac function in the chick embryo is also important because previous investigators have found that myocardial cell size and cardiac morphology are affected by a reduced incubation temperature. delaCruz et al. (5) observed a 4-fold increase in mortality and structural defects in $35 \%$ of embryos incubated at a temperature $2^{\circ} \mathrm{C}$ cooler than controls. Warbanow (18) studied the effect of incubation temperature on the ventricular muscle. He found an increase in ventricular muscle cell size in embryos incubated at a subnormal temperature.

Hypothermia produces changes in heart rate and blood pressure in the chick embryo. Cohn (2) demonstrated a direct relationship between temperature and heart rate in chick embryos in ovo. He speculated that heart rate was controlled through a temperature-dependent chemical reaction, but discussed neither the site of the control mechanism nor the effect on physiologic

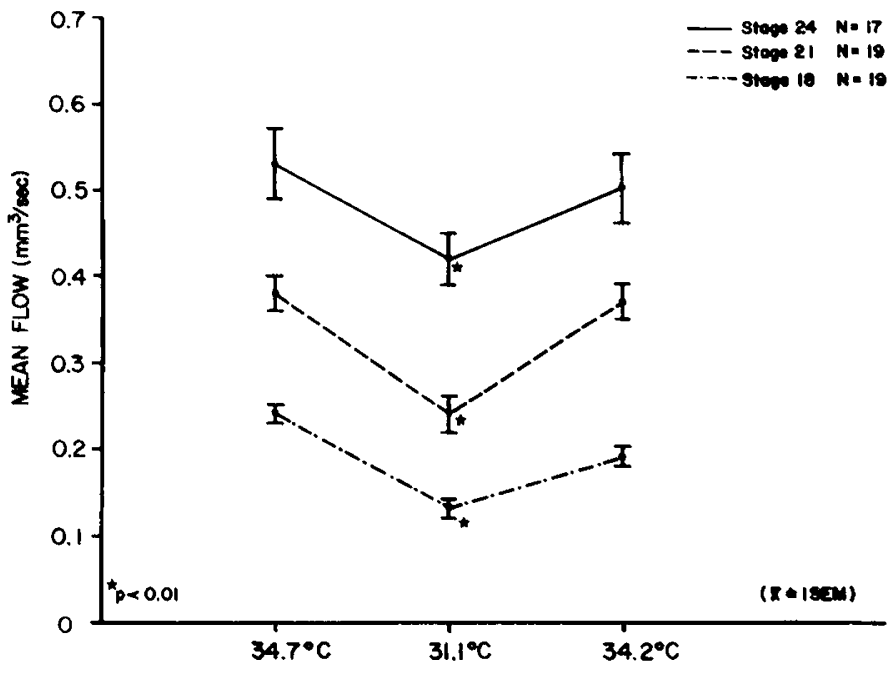

Fig. 3. Graph of mean dorsal aortic blood flow versus environmental temperature for experimental embryos at stages 18, 21, and 24.

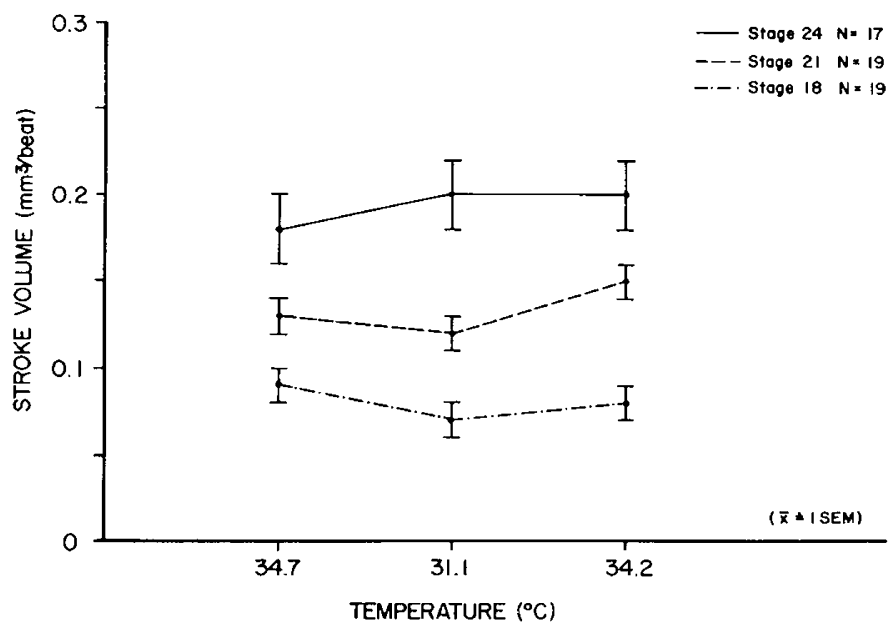

Fig. 4. Graph stroke volume index versus environmental temperature for experimental embryos at stages 18, 21 and 24 .

function in the embryo. There is an extensive literature demonstrating the direct relationship between heart rate and temperature in the chick embryo (14). The results of our study are in agreement with those previously published. Girard (8) investigated the effect of temperature on blood pressure in stage 18 embryos. Systolic blood pressure decreased slightly (less than 1 $\mathrm{mmHg}$ ) in response to a $15^{\circ} \mathrm{C}$ decrease in temperature. The 
blood pressure response to environmental cooling has not been measured in stage 21 or 24 chick embryos.

Our experiments are the first to demonstrate the effect of environmental hypothermia on an index of cardiac output in the embryo. There was a direct relationship among environmental temperature, heart rate, and dorsal aortic blood flow; however, stroke volume index remained constant. The stable stroke volume index suggests that embryonic myocardial function is not acutely affected by hypothermia because myocardial injury should result in a decrease in stroke volume and lag in the recovery period.

We conclude that hypothermic bradycardia is mediated by the myocardium. The response proceeds and is independent of functional autonomic innervation. Circulating neurohumoral agents may participate in the response to environmental cooling because receptors are present $(11,13)$. Heart rate and force of contraction in isolated chick myocardium are increased by adrenergic drugs but decreased by acetylcholine (13); however, these agents have not been isolated from the myocardium at early stages of development.

The effect of temperature on the cell membrane potential is a likely mechanism responsible for the bradycardia. Pacemaker cells located in the sinus venosus control the heart rate in the chick embryo (14). Coraboenf and Weidman (3) studied the relationship between temperature and intracellular action potential in calf Purkinje fibers. They observed a decrease in the rate of change of action potential with lower temperature. Phase four action potential is particularly depressed and markedly slows the heart rate. Sperelakis and Lehmkuhl (16) found that the change in membrane potential of spontaneously depolarizing cultured chick heart cells markedly slowed with cooling. Investigating this response further, Sperelakis and Lee (15) found that $\mathrm{Na}^{+}$and $\mathrm{K}^{+}$ ATPase activity was related directly to temperature. A temperature-affected decrease in the $\mathrm{Na}^{+} \mathrm{K}^{+}$pump and subsequent change in cell membrane action potential is the most likely mechanism for the reversible decrease in heart rate observed in our study.

Hypothermia is a common environmental risk for the developing chick because the hen leaves the nest for food and water. Bradycardia appears to be protective mechanism to compensate for temperature fluctuations, and may be the phylogenetic example of the bradycardia observed in mammals exposed to environmental hypothermia.

\section{REFERENCES AND NOTES}

1. Clark, E. B. and Hu, N.: Developmental hemodynamic changes in the chick embryo from stage 18 to 27 . Circ. Res., 51: 810 (1982).
2. Cohn, A. E.: Physiologic ontogeny: A. Chicken embryos. XIII. The temperature characteristic for the contraction rate of the whole heart. J. Gen. Physiol., 10: 369 (1927).

3. Coraboenf, E. and Weidman, S.: Temperature effects on electrical activity of Purkinje fibers. Helv. Physiol. Pharmacol. Acta, 12: 32 (1954).

4. Culver, N. G. and Fishman, D. A.: Pharmacological analysis of sympathetic function in the embryonic chick heart. Am. J. Physiol., 232: R116 (1977).

5. delaCruz, M., Campillo-Sainz, C., and Mundz-Armas, S.: Congenital heart defects in chick embryos subjected to temperature variations. Cir. Res., 18: 257 (1960).

6. Dudgeon, D. L., Randall, P. A., Hill, R. B., and McAfee, J. C.: Mild hypothermia: its effect on cardiac output and regional perfusion in the neonatal piglet. J. Pediatr. Surg., 15: 805 (1980)

7. Frieswick, G. M., Danielson, T., and Shideman, F. E.: Adrenergic inotropic responsiveness of embryonic chick and rat hearts. Dev. Neurosci., 2: 276 (1979).

8. Girard, H.: Arterial pressure in the chick embryo. Am. J. Physiol., 224: 454 (1973).

9. Hamburger, V. and Hamilton, H. L.: A series of normal stages in the development of the chick embryo. J. Morphol., 88: 49 (1951).

10. Higgins, D. and Pappano, A. J.: Developmental changes in the sensitivity of the chick embryo ventricle to Beta-adrenergic agonist during adrenergic innervation. Circ. Res., 48: 245 (1981).

11. Lipshultz, S., Shanfeld, J., and Chacko, S.: Emergence of beta-adrenergic sensitivity in the developing chicken heart. Proc. Natl. Acad. Sci., 78: 288 (1981).

12. Merrick, S. H., Hessel, E. A., and Dillard, D. H.: Determination of cardic output by thermodilution during hypothermia. Am. J. Cardiol., 46: 419 (1980).

13. Pappano, A. J.: Ontogenetic development of autonomic neuroaffector transmission and transmitter reactivity in embryonic and fetal hearts. Pharmacol. Rev., 29: 3 (1977).

14. Romanoff, A. L.: The Avian Embryo: Structure and Functional Development. (New York, MacMillan 1960).

15. Sperelakis, N. and Lee, E. C.: Characterization of $(\mathrm{Na}+, \mathrm{K}+)-\mathrm{ATPase}$ isolated from embryonic chick hearts and cultured chick heart cells. Biochem. Biophys. Acta, 233: 562 (1971).

16. Sperelakis, N. and Lehmkuhl, D.: Effect of temperature and metabolic poisons on membrane potentials of cultured heart cells. Am. J. Physiol., 213: 719 (1967).

17. Thauer, R.: The Circulation in Hypothermia of Nonhibernating Animals and Men. In: Handbook of Physiology, Section 2, Vol III. Ed: W. Hamilton and P. Dow. Washington, D.C., American Physiological Society, pp. 1899-1920 (1965).

18. Warbanow, W.: Contractility of the embryonic chick heart in hypothermiainduced cardiac hyperplasia and hypertrophy. Acta Biol. Med. Germ., 26 859 (1971)

19. We thank Dr. William Clarke for his help with the statistical analysis, Mrs. Sue Kucera for her patient work with the manuscript and Mr. Horst Jordan for graphics.

20. Dr. Clark is the recipient of NIH Research Career Development Award HD00376 and this study was supported by NIH Grant HD14723.

21. Requests for reprints should be addressed to: Dr. Edward B. Clark, Division of Pediatric Cardiology, Department of Pediatrics, University of Iowa Hospitais and Clinics, lowa City, Iowa 52242.

22. Received for publication December 29, 1982.

23. Accepted for publication May 20, 1983. 